\title{
Radiotherapy and high bilirubin may be metformin like effect on lung cancer via possible AMPK pathway modulation
}

\author{
Atasoy $\mathrm{O}^{1,2}$, Cini $\mathrm{N}^{1}$, Erdogan $\mathrm{MA}^{3}$, Yaprak $\mathrm{G}^{1}$, Erbas $\mathrm{O}^{2,4}$ \\ Izmir Katip Çelebi University, Faculty of Medicine, Department of Physiology, Izmir, Turkey. \\ alpero86@gmail.com
}

\begin{abstract}
PURPOSE: Life expectancy of cancer patients determine the regimen of treatment. There is no feasible marker that determines the survival other than the stage of the disease or other patients related factors. Bilirubin can be a revealing marker for these. The effect of bilirubin may be due to the fact that the genetic and biochemical processes of bilirubin also modulate the tumour microenvironment. Radiotherapy and bilirubin can produce an effect similar to metformin via AMPK pathway.

MATERIALS AND METHODS: This analysis was performed retrospectively in a cohort of 80 patients with a diagnosis of locoregional lung cancer with bilirubin levels in the accepted range. Receiver operating characteristic curve (ROC) analysis was performed to determine the optimal cut-off points. Pre-treatment serum total bilirubin (TBIL), direct bilirubin (DBIL), indirect bilirubin (IBIL) levels and tumour volumes in the prognosis of the patients were investigated.

RESULTS: The cut-off points for serum TBIL, DBIL and IBIL were $0.565 \mathrm{mg} / \mathrm{dL}, 0.105 \mathrm{mg} / \mathrm{dL}$ and $0.415 \mathrm{mg} / \mathrm{dL}$, respectively. High TBIL $47.5 \%$, high DBIL and high IBIL were observed in $45 \%$ of the entire patient population. The overall survival was three times longer in the high TBIL group than in the low TBIL group (OS; Hazard Ratio (HR), 0.33; 95\% Cl 0.16-0.70; $\mathrm{p}<0.001$ ), locoregional free survival (LRFS; HR, 0.44; 95\% Cl 0.27-0.71; $p<0.001$ ) and distant metastasis-free survival (DMFS; HR, 0.44; 95\% 0.25-0.80; $p<$ 0.001). Similarly, high DBIL and high IBIL levels have been associated with longer OS, LRFS, and DMFS with significant differences. In addition, in the survival analysis of the cohort stratified with gross tumour volume (GTV) $128.5 \mathrm{cc}$ and TBIL 0.565 cut-off values; In the comparison of high TBIL and low TBIL groups, a significantly longer OS was observed in the high TBIL group in the patients with a GTV volume greater than $128.5 \mathrm{cc}(\mathrm{p}<0.001)$.

CONCLUSION: Plasma bilirubin level at the time of diagnosis affects the survival of the patients independent of cancer stage and tumour volume. Possible additive interactions of radiotherapy and bilirubin are discussed with their pathophysiological mechanisms (Tab. 2, Fig. 7, Ref. 26). Text in PDF www.elis.sk

KEY WORDS: bilirubin, radiotherapy, AMPK, GTVcc, lung cancer.
\end{abstract}

\section{Introduction}

Lung cancer causes nearly $23 \%$ of cancer-related deaths in both sexes (1). The 5-year survival for all the subtypes of localized lung cancer is $59 \%$, this rate decreases to $31.7 \%$ for regional stage lung cancer (2). Less than half of the patients have localized disease at the time of diagnosis. The treatment of limited disease is mainly surgical, but in locoregional disease observed in approximately $30 \%$ of the patients, the treatment is chemo-radiotherapy (ChRT) $(2,3,4)$.

${ }^{1}$ Kartal City Hospital, Radiation Oncology Department, Istanbul, Turkey, ${ }^{2}$ Institute of Experimental Medicine, Tubitak Tekgeb, Gebze, Turkey, ${ }^{3}$ Izmir Katip Celebi University, Faculty of Medicine, Department of Physiology, Izmir, Turkey, and ${ }^{4}$ Demiroglu Bilim University, Faculty of Medicine, Department of Physiology, Istanbul, Turkey

Address for correspondence: M.A. Erdogan, Assoc Prof, DVM, PhD, Izmir Katip Celebi University, Faculty of Medicine, Department of Physiology, Izmir, Turkey.

Phone: +905433818677
Bilirubin is the natural end product of heme metabolism. Studies showed that total bilirubin (TBIL) and especially indirect bilirubin (IBIL) are associated with better survival in many diseases due to its antioxidant and antiproliferative effects through the biliverdin reductase cycle $(5,6)$. Studies have proven that increased bilirubin is associated with a better prognosis in cardiovascular diseases (7), autoimmune diseases (8), and cancer (9). In addition, it has been shown that the increased bilirubin in the healthy population is protective against many diseases with its high antiinflammatory and antioxidant properties (10). Serum bilirubin as part of normal heme catabolism plays an important role against oxidative stress. Therefore, uridine diphosphate - glucuronosyl - transferase 1-1 (UGT1A1) gene polymorphisms in this pathway may protect against cancer formation caused by oxidative stress. It has been observed that people with low bilirubin levels are more often diagnosed with lung cancer (11). Contrary to this effect, the antioxidant activity mechanism of bilirubin may have negative effect during treatment. With its antioxidant effect, it can protect cancerous cells from the cytotoxic effects of chemotherapy 


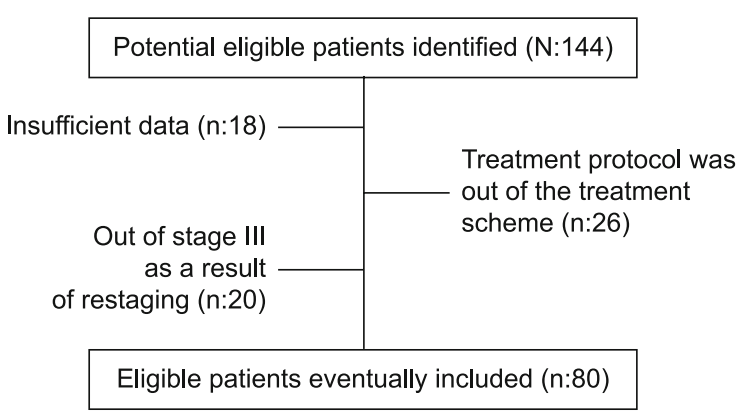

Fig. 1. Patient elimination chart of the study.

(ChT) and radiotherapy (RT). And it can also reduce the response to treatment by reversing the production of reactive oxygen radicals, which are the products of the indirect damage mechanism of $\mathrm{RT}$ and induce cancer death. In addition, it is known that bilirubin is a T lymphocyte inhibitor $(12,13)$. Even though many different effects have been proven by previous studies, the direction of the mechanism of high bilirubin levels on cancer and its treatment in oncological patients is still unclear.

Adenosine monophosphate-activated kinase (AMPK) is an evolutionarily conserved cellular energy sensor that belongs to the serine-threonine kinase enzyme family and is found in almost all eukaryotic cells. Activation of the AMPK pathway increases in cellular stress situations. It is known that in individuals with high bilirubin levels due to the UGT1A1 polymorphism, AMPK activation increases more than in the normal population in cases of induced stress (14).

In our study, we hypothesized that high bilirubin levels through the AMPK pathway both limit tumour growth and cause a better tumour response by converting treatment-induced stress in favour. Such a relation between these variables has been considered for the first time in literature. For this purpose, locally advanced stage patients, who received ChRT as standard therapy were included in our study. All of the patients were Stage III and received concomitant ChT treatment with standard 60 Gy RT. Since the bilirubin levels may be affected by the given treatments, the TBIL, IBIL, and DBIL levels were measured at the time of diagnosis.

Tab. 1. Patients characteristics.

\begin{tabular}{|c|c|c|c|c|c|c|c|c|c|}
\hline & & BIL $(0.565$ & & DBIL & $0.105)$ & & & $\operatorname{VBIL}(0.41$ & \\
\hline & $\begin{array}{c}\text { HİGH } \\
36(\% 45)\end{array}$ & $\begin{array}{c}\text { LOW } \\
44(\% 55)\end{array}$ & $\mathrm{p}$ & $\begin{array}{c}\text { HİGH } \\
38(\% 47.5)\end{array}$ & $\begin{array}{c}\text { LOW } \\
42(\% 52.5)\end{array}$ & $\mathrm{p}$ & $\begin{array}{c}\text { HİGH } \\
36(\% 45)\end{array}$ & $\begin{array}{c}\text { LOW } \\
44(\% 55)\end{array}$ & $\mathrm{p}$ \\
\hline $\begin{array}{l}\text { Age } \\
<65 \\
>65\end{array}$ & $\begin{array}{l}17(21.3) \\
19(23.8)\end{array}$ & $\begin{array}{l}21(26.3) \\
23(28.7)\end{array}$ & 0.964 & $\begin{array}{l}19(23.8) \\
19(23.8)\end{array}$ & $\begin{array}{l}19(23.8) \\
23(28.7)\end{array}$ & 0.67 & $\begin{array}{l}18(22.5) \\
18(22.5)\end{array}$ & $\begin{array}{l}20(25) \\
24(30)\end{array}$ & 0.685 \\
\hline $\begin{array}{l}\text { Gender } \\
\text { Female } \\
\text { Male }\end{array}$ & $\begin{array}{c}6(7.5) \\
30(37.5)\end{array}$ & $\begin{array}{c}4(5) \\
40(50)\end{array}$ & 0.308 & $\begin{array}{c}5(6.3) \\
33(41.3)\end{array}$ & $\begin{array}{c}5(6.3) \\
37(46.2)\end{array}$ & 0.866 & $\begin{array}{c}6(7.5) \\
30(37.5)\end{array}$ & $\begin{array}{c}4(5.0) \\
40(50.0)\end{array}$ & 0.308 \\
\hline $\begin{array}{l}\text { Pathology } \\
\text { Nonsmall Cell } \\
\text { Squamous } \\
\text { Adenocarcinoma } \\
\text { Large Cell } \\
\text { Small Cell } \\
\end{array}$ & $\begin{array}{c}26(32.5) \\
17(21.3) \\
8(10) \\
1(1.3) \\
10(12.5)\end{array}$ & $\begin{array}{l}35(43.8) \\
20(25) \\
12(15) \\
3(3.8) \\
9(11.3) \\
\end{array}$ & 0.727 & $\begin{aligned} & 28(35) \\
& 17(21.3) \\
& 10(12.5) \\
& 1(1.3) \\
& 10(12.5)\end{aligned}$ & $\begin{array}{l}33(41.3) \\
20(25) \\
10(12.5) \\
3(3.8) \\
9(11.3) \\
\end{array}$ & 0.777 & $\begin{array}{c}16(20) \\
8(10) \\
2(2.5) \\
10(12.5) \\
\end{array}$ & $\begin{array}{c}21(26.3) \\
12(15) \\
2(2.5) \\
9(11.3) \\
\end{array}$ & 0.865 \\
\hline $\begin{array}{c}\text { T stage } \\
\text { T1 } \\
\text { T2 } \\
\text { T3 } \\
\text { T4 } \\
\end{array}$ & $\begin{array}{c}5(6.3) \\
8(10) \\
15(18.8) \\
8(10) \\
\end{array}$ & $\begin{array}{c}4(5) \\
10(12.5) \\
15(18.8) \\
15(18.8) \\
\end{array}$ & 0.641 & $\begin{array}{c}5(6.3) \\
8(10) \\
15(18.8) \\
10(12.5) \\
\end{array}$ & $\begin{array}{c}4(5.0) \\
10(12.5) \\
15(18.8) \\
13(16.3) \\
\end{array}$ & 0.913 & $\begin{array}{c}4(5.0) \\
9(11.3) \\
14(17.5) \\
9(11.3) \\
\end{array}$ & $\begin{array}{c}5(6.3) \\
9(11.3) \\
16(20) \\
14(17.5) \\
\end{array}$ & 0.911 \\
\hline $\begin{array}{c}\text { N stage } \\
\text { N1 } \\
\text { N2 } \\
\text { N3 } \\
\end{array}$ & $\begin{array}{c}8(10) \\
18(22.5) \\
10(12.5) \\
\end{array}$ & $\begin{array}{l}10(12.5) \\
17(21.3) \\
17(21.3) \\
\end{array}$ & 0.528 & $\begin{array}{c}7(8.8) \\
17(21.3) \\
14(17.5) \\
\end{array}$ & $\begin{array}{l}11(13.7) \\
18(22.5) \\
13(16.3) \\
\end{array}$ & 0.685 & $\begin{array}{c}8(10) \\
19(23.8) \\
9(11.3) \\
\end{array}$ & $\begin{array}{c}10(12.5) \\
16(20) \\
18(22.5) \\
\end{array}$ & 0.258 \\
\hline $\begin{array}{l}\text { TNM stage } \\
\text { IIIA } \\
\text { IIIB } \\
\text { IIIC }\end{array}$ & $\begin{array}{c}13(16.3) \\
17(21.3) \\
6(7.5)\end{array}$ & $\begin{array}{l}13(16.3) \\
18(22.5) \\
13(16.3)\end{array}$ & 0.401 & $\begin{array}{c}12(15) \\
17(21.3) \\
9(11.3)\end{array}$ & $\begin{array}{l}14(17.5) \\
18(22.5) \\
10(12.5)\end{array}$ & 0.983 & $\begin{array}{c}14(17.5) \\
16(20) \\
6(7.5)\end{array}$ & $\begin{array}{c}12(15) \\
19(23.8) \\
13(16.3)\end{array}$ & 0.331 \\
\hline $\begin{array}{r}\text { GTV Volume } \\
\quad<128.55 \mathrm{cc} \\
>128.55 \mathrm{cc} \\
\end{array}$ & $\begin{array}{c}27(33.8) \\
9(11.3)\end{array}$ & $\begin{array}{l}23(28.7) \\
21(26.3)\end{array}$ & 0.037 & $\begin{array}{c}26(32.5) \\
12(15)\end{array}$ & $\begin{array}{c}24(30) \\
18(22.5)\end{array}$ & 0.298 & $\begin{array}{c}27(33.8) \\
9(11.3)\end{array}$ & $\begin{array}{l}23(28.7) \\
21(26.3)\end{array}$ & 0.037 \\
\hline $\begin{array}{l}\text { Chemotherapy } \\
\text { Cisplatin+etoposide } \\
\text { Carboplatin+paclitaxel } \\
\text { Carboplatin+etoposide } \\
\text { Cisplatin+paclitaxel }\end{array}$ & $\begin{array}{c}14(17.5) \\
21(26.2) \\
0(0) \\
1(1.3)\end{array}$ & $\begin{array}{c}14(17.5) \\
26(32.5) \\
2(2.5) \\
2(2.5)\end{array}$ & 0.555 & $\begin{array}{c}14(17.5) \\
23(28.7) \\
0(0) \\
1(1.3)\end{array}$ & $\begin{array}{c}14(17.5) \\
24(30) \\
2(2.5) \\
2(2.5)\end{array}$ & 0.54 & $\begin{array}{c}15(18.8) \\
20(25) \\
0(0) \\
1(1.3)\end{array}$ & $\begin{array}{c}13(16.3) \\
27(33.8) \\
2(2.5) \\
2(2.5)\end{array}$ & 0.432 \\
\hline
\end{tabular}

TNM stage - tumor-node-metastasis stage. TBIL - total bilirubin. DBIL - direct bilirubin. INBIL - indirect bilirubin. GTV - gross tumor volume 


\section{0-109}

\section{Material and methods}

\section{Patients and methods}

In this analysis, a cohort of 144 patients, who were admitted to Kartal City Hospital between July 2015 and January 2020 with the diagnosis of non-small cell lung cancer (NSCLC) and small cell lung cancer (SCLC) and received concurrent ChRT was retrospectively analysed. All the patients were reclassified according to the 8 th The American Joint Committee on Cancer (AJCC) classification system (15) Potentially eligible patients had to have pathologically confirmed stage IIIA-IIIB-IIIC lung cancer without prior treatment and complete concomitant ChT with 60 Gy RT as standard therapy. Patients with a diagnosis of another known malignancy and high bilirubin levels due to another disease were not included in the study.

Of the 144 patients; 18 patients were excluded from the study because their bilirubin levels were not checked before treatment or at the time of diagnosis, 26 patients were excluded because the treatment protocol was out of the treatment scheme specified for our study or could not complete the treatment, and 20 patients were excluded because they were out of Stage III as the result of restaging. Thus, the analysis continued with 80 patients, who were eligible for the conditions of our study (Fig. 1).

Information was collected on individual patient records from electronic and papery, and information about survival was obtained from the follow-up registry of Kartal City Hospital. The informa- tion collected included age, sex, pathology, time of diagnosis, time of death/relapse/metastasis, ChT regime and pre-treatment serum TBIL, direct bilirubin (DBIL) and IBIL levels. All biochemical analyses were performed in the Department of Biochemistry Laboratory, Kartal City Hospital. We also excluded patients with TBIL levels lower than $0.15 \mathrm{mg} / \mathrm{dL}$ and higher than $1.35 \mathrm{mg} / \mathrm{dL}$ for men and women. To avoid the potential impact of surgery or ChT on bilirubin level, we ensured that each eligible patient had a pre-treatment biochemical test.

\section{Statistical analysis}

The receiver operating characteristic (ROC) curve analysis was performed to evaluate the ability of TBIL, DBIL and IBIL levels to predict long-term outcomes and to determine the optimal cut-off points. The cut-off points with the highest sum of sensitivity and specificity were determined that dichotomize the entire cohort into two. A Chi-square test was used to compare categorical variables.

The following endpoints were evaluated: overall survival (OS), defined as the interval from the time of being diagnosed to the time of death; locoregional relapse-free survival (LRFS) defined as the interval from the time of being diagnosed to the development of regional or locoregional relapse/progression of the disease and distant metastasis-free survival (DMFS), defined as the interval from the time of being diagnosed to the time of diagnosis of distant metastases.

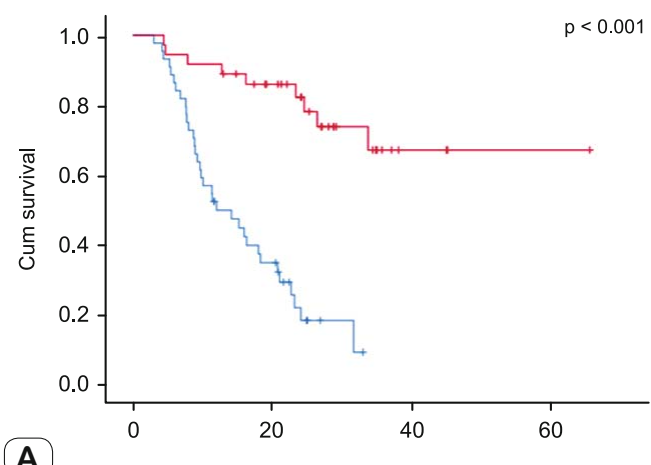

A

Overall survival

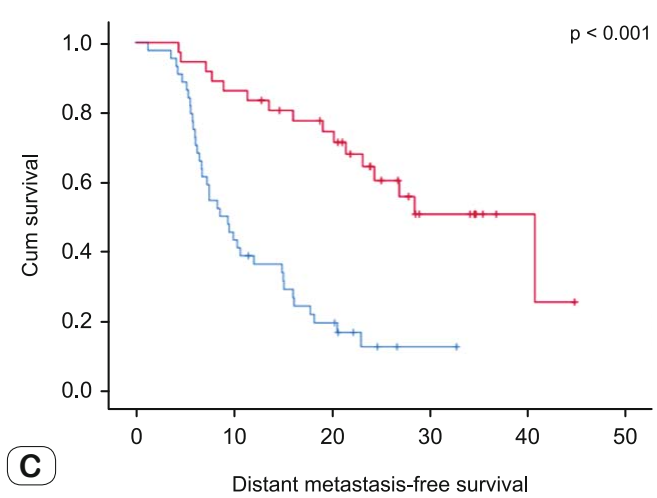

Distant metastasis-free surviva

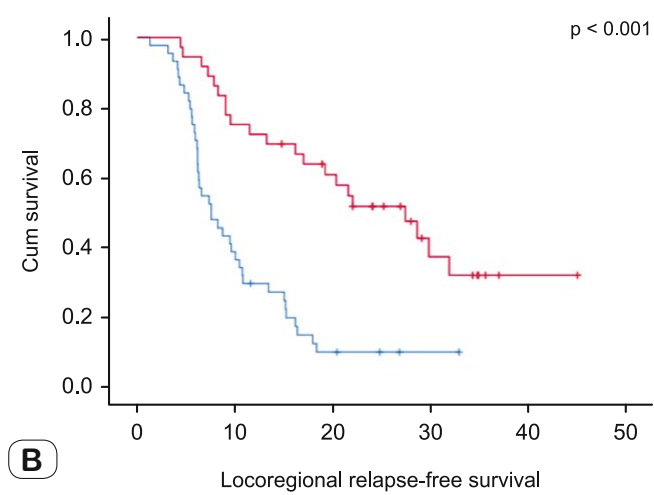

TBIL

$\neg>0.565$

$\neg>0.565$

$+<0.565-$ censored

$+>0.565-$ censored

Fig. 2. Kaplan-Meier curves for (A) overall survival, (B) locoregional relapse-free survival, and (C) distant metastasis-free survival stratified by total bilirubin (TBIL) level. 


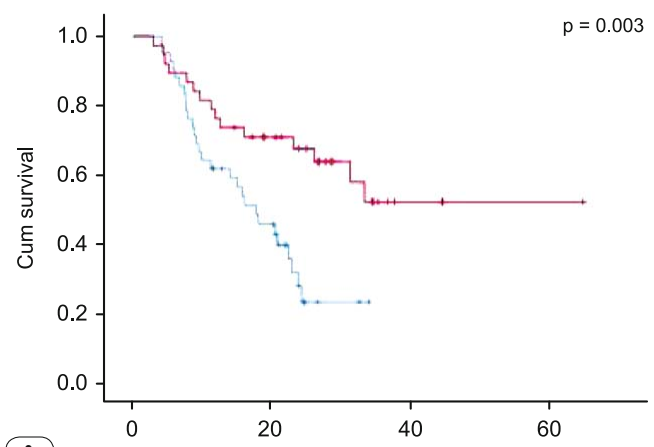

A

Overall survival

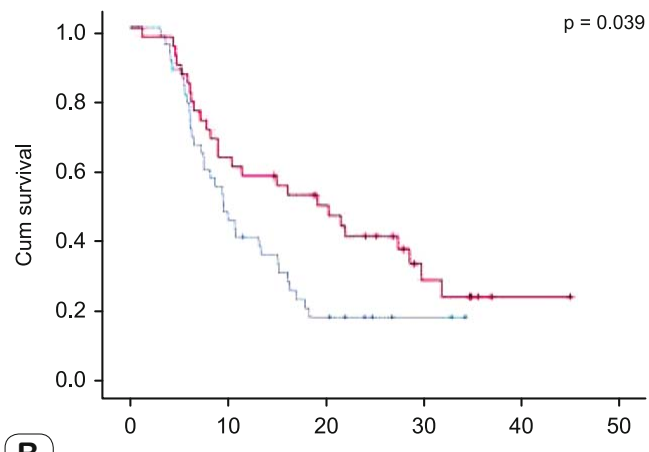

B

Locoregional relapse-free survival

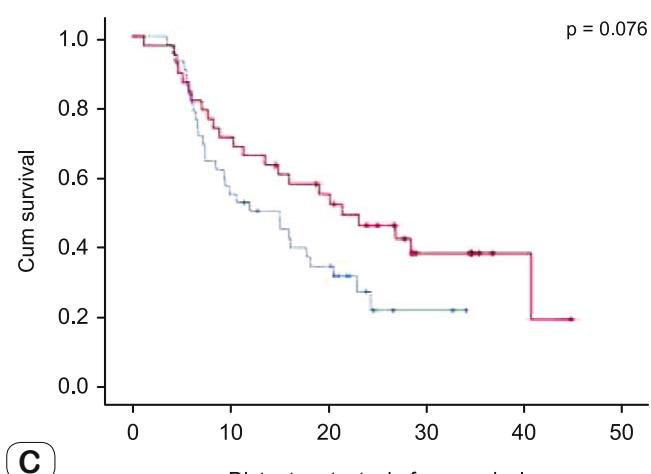

DBIL

$\neg<0.105$

$\neg>0.105$

$+<0.105-$ censored

$+>0.105-$ censored

\section{C}

Distant metastasis-free survival

Supplementary Figure 1. Kaplan-Meier curves for (A) overall survival, (B) locoregional relapse-free survival, and (C) distant metastasis-free survival stratified by direct bilirubin (DBIL) level.

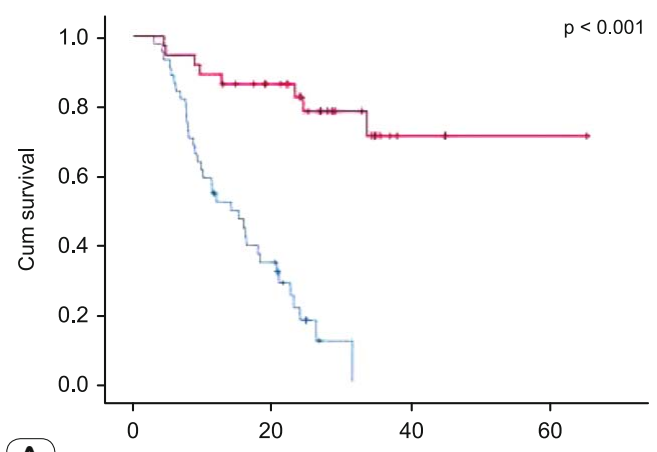

A

Overall survival

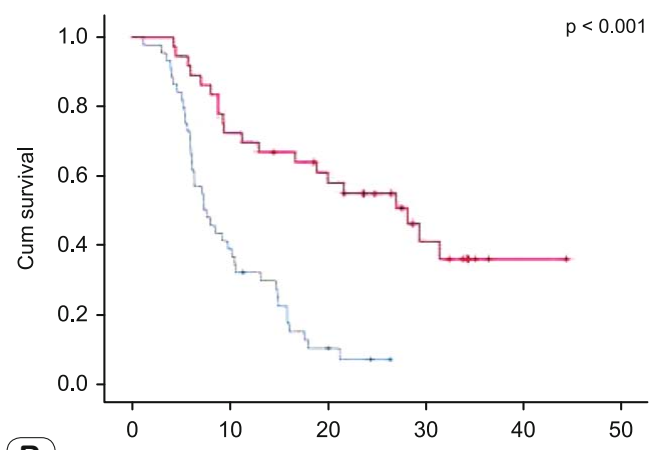

(B)

Locoregional relapse-free survival

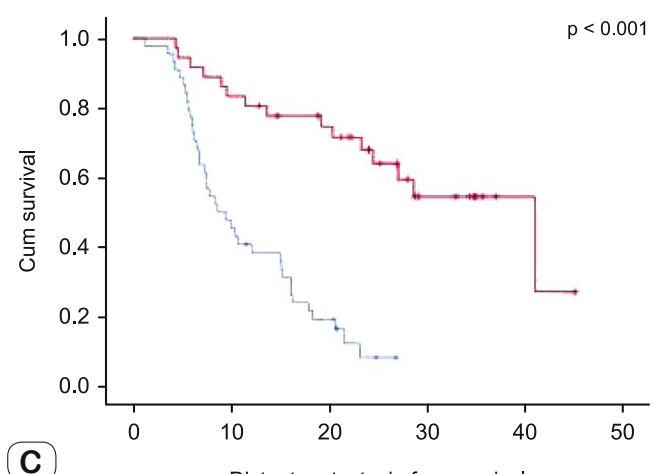

IBIL

$\neg<0.415$

$+<0.415-$ censored

$+>0.415-$ censored

\section{C}

Distant metastasis-free survival

Supplementary Figure 2. Kaplan-Meier curves for (A) overall survival, (B) locoregional relapse-free survival, and (C) distant metastasis-free survival stratified by indirect bilirubin (IBIL) level. 


\section{0-109}

Kaplan-Meier curves were drawn for these endpoints and differences were compared by the log-rank test. To perform multivariate analyses, the Cox proportional hazards model was used. In univariate analyses variables reaching $p=0.1$ as a significant level sug gested a trend and were included in multivariate analysis. $p$ values of less than 0.05 were considered statistically significant. All analyses were performed using SPSS 15.0 software (SPSS, Inc., Chicago, IL, USA).

\section{Results}

\section{Patient population characteristics}

A total of 80 patients with stage IIIA-IIIB-IIIC lung cancer, who received concurrent ChRT were included in our study. Table 1 shows the main characteristics of the patient population included in the study.

The OS, LRFS and DMFS cut-off points for TBIL were determined as $0.565 \mathrm{mg} / \mathrm{dl}, 0.575 \mathrm{mg} / \mathrm{dl}$ and $0.575 \mathrm{mg} / \mathrm{dl}$, respectively. In survival analysis, the cut-off point for TBIL for OS was chosen as a uniform point of $0.565 \mathrm{mg} / \mathrm{dl}$. Similarly, a level of $0.105 \mathrm{mg} / \mathrm{dl}$ for DBIL and a level of $0.415 \mathrm{mg} / \mathrm{dl}$ for IBIL were chosen as cutoff points for survival analysis of OS. In the entire patient population, $45 \%$ high TBIL level, $47 \%$ high TBIL level and $45 \%$ high IBIL level were observed. Chi-square analysis performed between high versus low bilirubin levels in all variables and significant $\mathrm{p}$ values observed only for TBIL and IBIL in Gross Tumour Volume cc (GTVcc). All of the high-TBIL group and the high-IBIL group patients significantly had smaller GTVec (TBIL $p=0.037$ and IBIL $p=0.037)$. No statistically significant difference was found in terms of other variables except GTVcc (Tab. 1).

\section{Survival analysis}

From 80 patients for the entire cohort; LR developed in 60 patients, while LRFS duration was 15.0 months (95\% CI 12.8-17.3), 53 patients developed distant metastasis, while DMFS was 16.7 months (95\% CI 14.4-17.3), and 43 people died that had an average OS of 19.6 months (95\% CI 17.1-22.2).

High TBIL group had significantly prolonged OS compared to low TBIL group, median OSS was 50.2 months versus 15.9 months (Hazard Ratio (HR), 0.33; 95\% CI 0.16-0.70; p < 0.001) (Fig. 2A).

Among patients with high TBIL and low TBIL, the 2-year OS rates were $\% 72.1$ and $18.1 \%$, respectively. Also, LRFS (HR, 0.44; 95\% CI 0.27-0.71; $<<0.001$ ) (Fig. 2B) and DMFS (HR, 0.44; 95\% $0.25-0.80 ; \mathrm{p}<0.001$ ) (Fig. 2C) development time was significantly longer in the patients of the high TBIL group. The 2-year LRFS and DMFS development rates were $54.1 \%$ and $56.7 \%$ for the patients in the high TBIL group and $10.3 \%$ and $14.9 \%$ for the patients in the low TBIL group, respectively.

Similarly, the high DBIL group had a significantly longer OS (HR, 0.46; 95\% CI 0.25-86; $p=0.003$ ) (Supplementary Figure 1A), LRFS (HR, 0.66; 95\% CI 0.43-1.03; p = 0.039) (Supplementary Figure 1B) and DMFS (HR, 0.71; 95\% CI 0.44-1.13; p
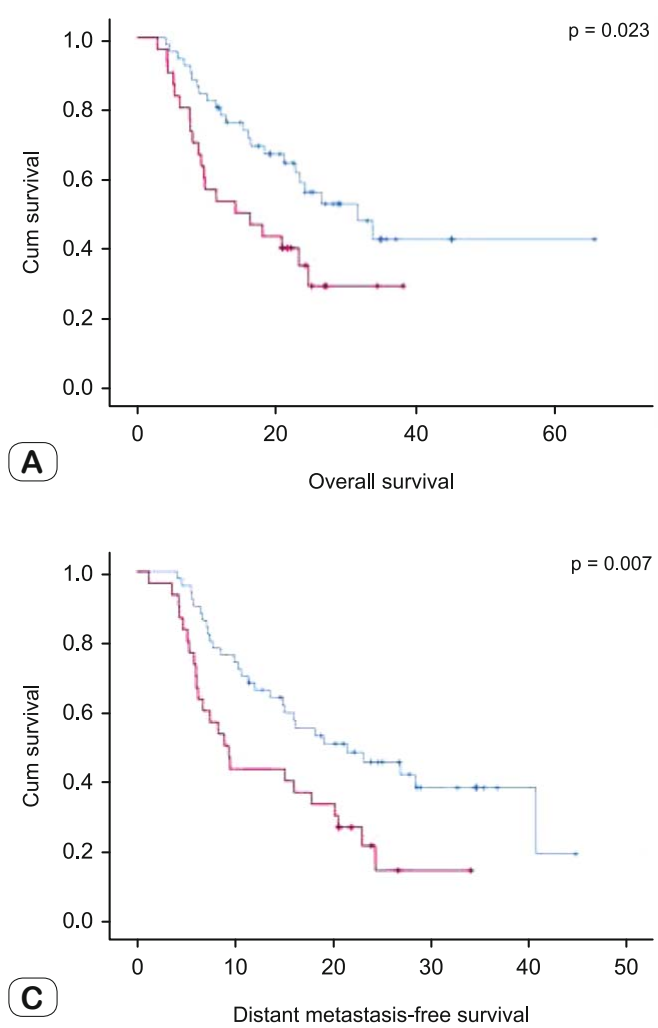

Distant metastasis-free surviva

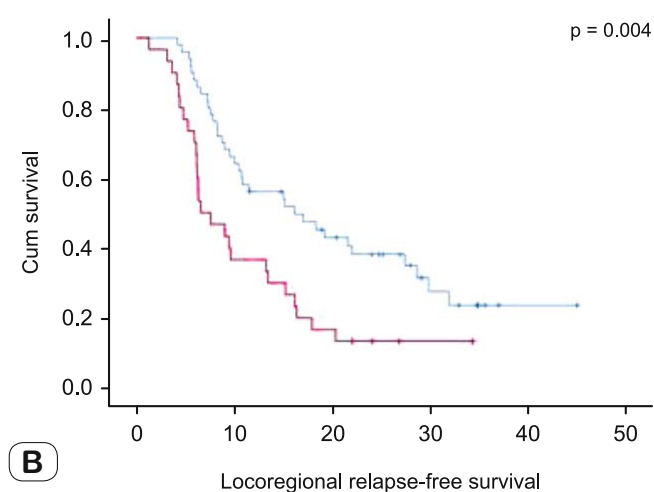

GTVcc

$\neg<128.55$

$\neg>128.55$

$+<128.55-$ censored

$+>128.55-$ censored

Supplementary Figure 3. Kaplan-Meier curves for (A) overall survival, (B) locoregional relapse-free survival, and (C) distant metastasis-free survival stratified by GTV cc volume. 

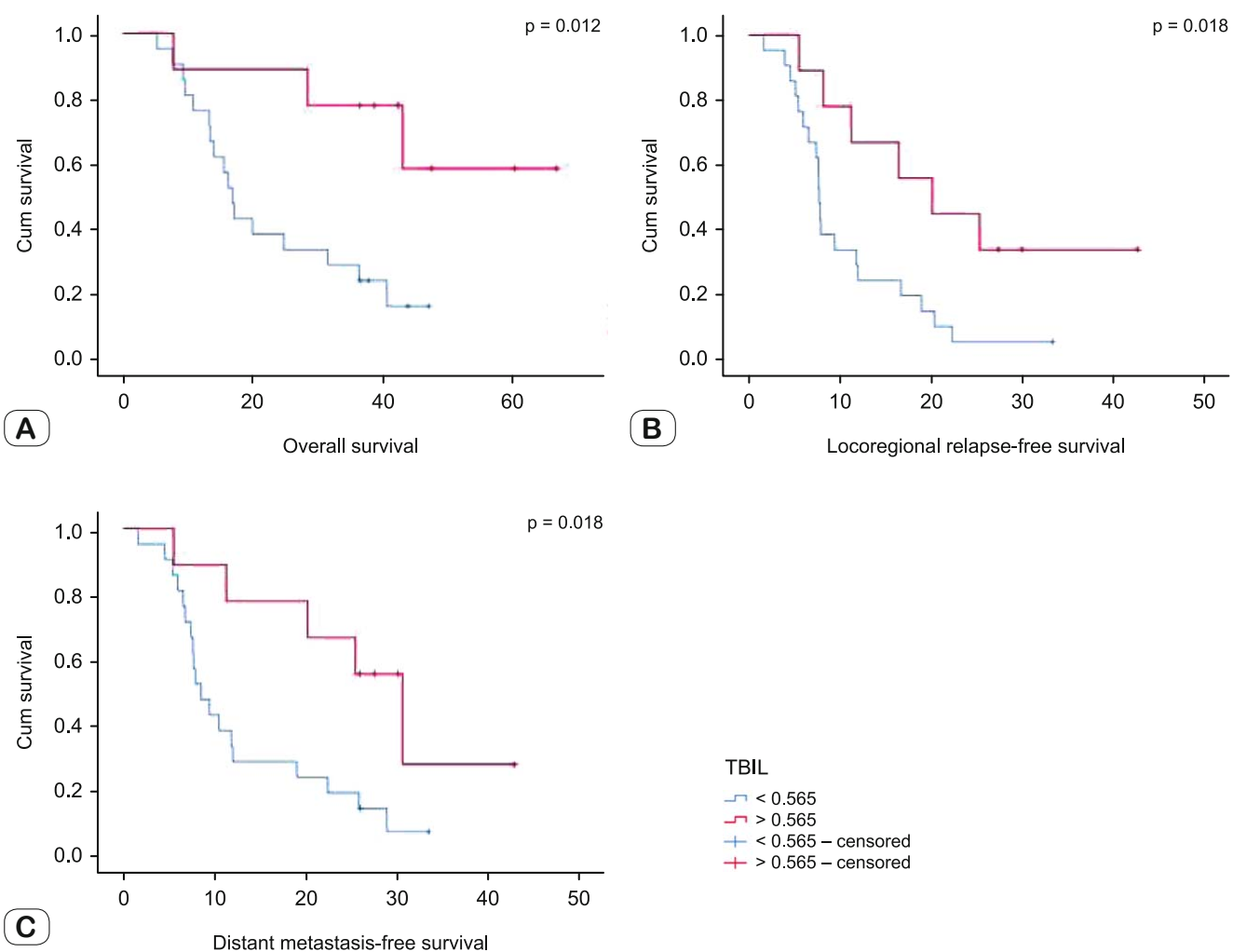

Distant metastasis-free survival

Supplementary Figure 4. Kaplan-Meier curves for (A) overall survival, (B) locoregional relapse-free survival, and (C) distant metastasis-free survival stratified by total bilirubin (TBIL) that with stratified by GTVec.

$=0.076$ ) (Supplementary Figure 1C) than the low DBIL group. The 2-year OS, LRFS, and DMFS were $22.8 \%, 69.5 \%$, and $43.1 \%$ for the patients in the High DBIL group and $19.2 \%, 21.5 \%$, and $22.1 \%$ for the patients in the low DBIL group, respectively.

The high IBIL group had longer OS (HR, 0.32; 95\% CI 0.15$0.68 ; \mathrm{p}<0.001$ ) (Supplementary Figure 2A), LRFS (HR, 0.41; 95\% CI $0.25-0.67 ; \mathrm{p}<0.001$ ) (Supplementary Figure 2B) and DMFS (HR, 0.41; 95\% CI 0.74-1.30; p <0.001) (Supplementary Figure 2C) with significant difference. The 2-year OS, LRFS and DMFS values were $73.2 \%, 58.3 \%$ and $63.2 \%$ for the patients in the high IBIL group and $10.2 \%, 9.0 \%$, and $10.1 \%$ for the low IBIL group, respectively.

Also examined the survival analysis of GTV volume, which is the only significant factor between high and low bilirubin levels in the Chi-square analysis. For the OS, survival was significantly prolonged to 37.8 months for the patients with GTV cc volume less than $128.5 \mathrm{cc}$ (HR 0.54; 95\% CI 0.30-0.86; p=0.023) (Supplementary Figure 3A). The LRFS time is longer in patients with low GTVec volumes (HR 0.58; 95\% CI 0.32-0.95; p <0.001) (Supplementary Figure 3B), likewise DMFS time (HR 0.61; 95\% CI 0.36-0.92; $\mathrm{p}=0.007$ ) (Supplementary Figure 3C).

Survival analysis for TBIL stratified with GTVcc; comparing low and high TBIL levels for the patients with high GTVcc volume for OS, significantly longer survival was observed in the high TBIL group as 29,1 months for High TBIL and 13,12 months for low TBIL group (HR 0.47; 95\% CI 0.12-1.57; $\mathrm{p}=$ 0.012 ) (Supplementary Figure 4A). The same was observed for LRFS (HR 0.47; 95\% CI 0.21-0.96; $\mathrm{p}=0.018$ ) (Supplementary Figure 4B) and DMFS (HR 0.50; 95\% CI 0.18-1.24; $\mathrm{p}=0.018$ ) (Supplementary Figure 4C).

To verify survival outcomes, we also re-performed the Kaplan-Meier analysis of the entire cohort stratified by another median serum TBIL level of $0.605 \mathrm{mg} / \mathrm{dL}$. The results showed that patients with TBIL levels higher than $0.605 \mathrm{mg} / \mathrm{dL}$ had prolonged OS, LRFS, and DMFS ( $<<0.001, \mathrm{p}<0.001, \mathrm{p}<0.001$, respectively) (Supplementary Figure 5A-C).

\section{Univariate analysis and multivariate analysis of OS}

In the univariate analysis age, gender, Tumor (T) stage, Node (N) stage, Tumor-Node-Metastasis stage (TNM) stage, GTVcc, pathology type, ChT regime, TBIL level, DBIL level and IBIL level were determined as important potential prognostic factors for OS. Variables that provide the determined $\mathrm{p}=0.1$ significance trend with univariate analysis, were analysed with multivariate analysis for OS. Gross Tumor Volume $c c(p=0.026)$, TBIL level $(p=0.000)$, DBIL level $(p=0.004)$, and IBIL level $(p=0.000)$ had significant $p$-values and HR's indicating that they are independent, significant predictors for OS. High serum TBIL, DBIL, IBIL levels, and GTV cc were positive prognostic factors (Tab. 2). 
100-109

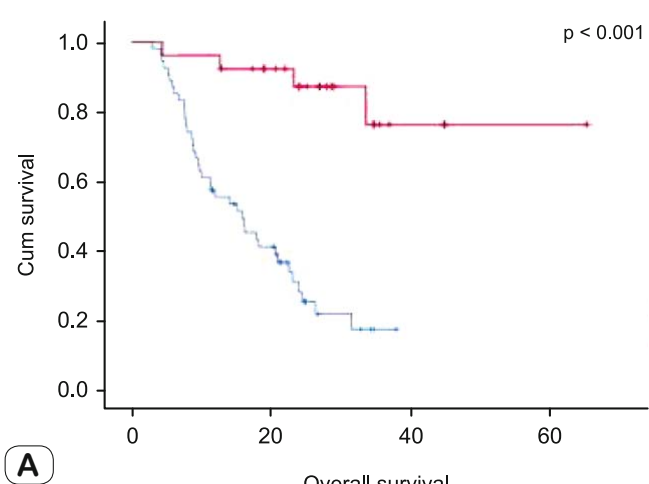

(A)

Overall survival

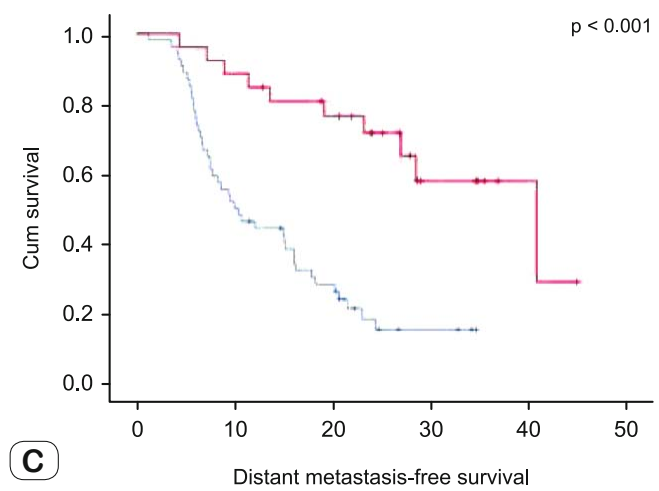

\section{C}

Distant metastasis-free survival

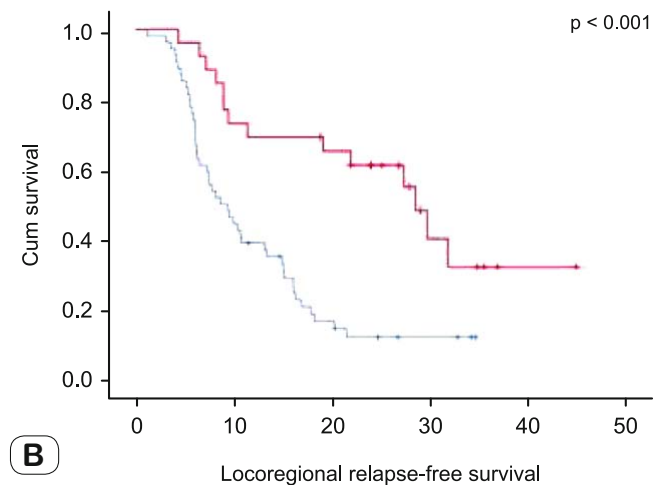

TBIL

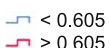

$\neg>0.605$

$+<0.605-$ censored

$+>0.605-$ censored

Supplementary Figure 5. Kaplan-Meier curves for (A) overall survival, (B) locoregional relapse-free survival, and (C) distant metastasis-free survival stratified by another median serum TBIL level of $0.605 \mathrm{mg} / \mathrm{dL}$.

\section{Discussion}

The antioxidant and anti-tumour effects of bilirubin have been reported in literature. Our hypothesis is among the firsts in the literature that links both high bilirubin levels and RT through the AMPK pathway with a good prognosis in lung cancer.

In this study, prognostic factors affecting bilirubin levels and the effects of bilirubin levels on the survival times in the patients with locoregional stage lung cancer, with a low survival rate, were investigated and tried to be explained. In our statistical and survival analysis, some significant differences were observed in the high bilirubin level groups compared to the low bilirubin groups. Significantly longer durations of OS, LRFS, and DMFS were observed in the patients with high TBIL, DBIL and IBIL levels compared to the patients with low TBIL, DBIL, and IBIL levels. In addition to high TBIL, IBIL and DBIL levels, independent of all other prognostic factors, GTV cc volume has been observed to be an important prognostic predictor factor for OS ( $p<0.001$, $\mathrm{p}<0.001, \mathrm{p}=0.004$ and $\mathrm{p}=0.026$ respectively).

In the similar study, $\mathrm{Li}$ et al showed the effect of bilirubin levels on survival times that; in 1617 patients with resectable lung cancer, preoperative bilirubin levels were collected retrospectively, and OS, disease-free survival and distant-metastasisfree survival times were significantly longer at slightly increased TBIL ( $>9.50 \mathrm{mmol} / \mathrm{L})$, IBIL $(>6.95 \mathrm{mmol} / \mathrm{L})$ and DBIL $(>3.45$ $\mathrm{mmol} / \mathrm{L}$ ) levels, and IBIL levels have been shown to be a prognostic predictor (16).

The TNM staging system is accepted as the most determining factor for the survival outcomes in the patients with lung cancer. Although all the patients included in our study were stage III, they were in different $\mathrm{T}$ and $\mathrm{N}$ stages. Stage III lung cancer represents a heterogeneous population; even though they are in the same TNM stage there are differences in survival outcomes between the patients receiving curative ChRT. This information suggests that, beyond the TNM staging, a detailed pre-treatment evaluation may improve the clinical outcome of the patients. In the study Jeremic et al (17) investigated the prognostic factors and proved that factors such as: female gender, lower KPS, less pronounced weight loss, lower stage, and squamous histology predict better OS and local PFS regardless of treatment.

In our study, besides TBIL, IBIL and DBIL levels and GTVcc volume; Age, gender, histology subtype, T stage, N stage, TNM stage, ChT regimen were identified as other potential prognostic factors and included in the survival analysis. It was observed that the $\mathrm{T}$ stage, the $\mathrm{N}$ stage, and the TNM stage had no effect on survival as prognostic factors in all stage III patients (T stage $\mathrm{p}$ : 0.809 , N stage $\mathrm{p}: 0.621$, TNM stage $\mathrm{p}=0.640$ ).

To determine the relationship with the survival time, the cutoff value for GTVcc was calculated as $128.55 \mathrm{cc}$ considering the sensitivity and specificity rates of the analysis. In the survival 
Tab. 2. Univariate and multivariate analysis for overall survival.

\begin{tabular}{|c|c|c|c|c|}
\hline & Univariate & & Multivariate & \\
\hline & Hazard ratio $(95 \% \mathrm{CI})$ & $\mathrm{P}$ & Hazard ratio $(95 \% \mathrm{CI})$ & $\mathrm{P}$ \\
\hline Age & & 0.126 & & \\
\hline$<65$ & reference & & & \\
\hline$>65$ & $2.007(0.82-4.90)$ & & & \\
\hline Gender & & 0.121 & & \\
\hline Female & reference & & & \\
\hline Male & $0.321(0.08-1.35)$ & & & \\
\hline Pathology & & 0.269 & & \\
\hline Small Cell & reference & & & \\
\hline Nonsmall Cell & & & & \\
\hline Squamous & $2.816(0.89-8.85)$ & & & \\
\hline Adenocarcinoma & $1.714(0.48-6.16)$ & & & \\
\hline Large Cell & $5.143(0.48-6.16)$ & & & \\
\hline T stage & & 0.809 & & \\
\hline $\mathrm{T} 1$ & reference & & & \\
\hline $\mathrm{T} 2$ & $1.563(0.31-7.82)$ & & & \\
\hline T3 & $1.25(0.28-5.59)$ & & & \\
\hline $\mathrm{T} 4$ & $1.944(0.41-9.24)$ & & & \\
\hline N stage & & 0.621 & & \\
\hline N1 & reference & & & \\
\hline N2 & $1.48(0.47-4.65)$ & & & \\
\hline N3 & $1.82(0.55-6.07)$ & & & \\
\hline TNM stage & & 0.64 & & \\
\hline IIIA & reference & & & \\
\hline IIIB & $1.556(0.56-4.32)$ & & & \\
\hline IIIC & $1.604(0.49-5.3)$ & & & \\
\hline GTV Volume & & 0.075 & & 0.026 \\
\hline$<128.55 \mathrm{cc}$ & reference & & reference & \\
\hline$>128.55 \mathrm{cc}$ & $2.348(0.92-6.02)$ & & $1.984(1.09-3.63)$ & \\
\hline Chemotherapy & & 0.912 & & \\
\hline Cisplatin+etoposide & reference & & & \\
\hline Carboplatin+paclitaxel & $1.073(0.42-2.75)$ & & & \\
\hline Carboplatin+etoposide & $0.867(0.05-15.3)$ & & & \\
\hline Cisplatin+paclitaxel & $0.433(0.04-5.35)$ & & & \\
\hline Total bilirubin & & 0.000 & & 0.000 \\
\hline High & reference & & reference & \\
\hline Low & $0.098(0.04-0.28)$ & & $0.148(0.07-0.33)$ & \\
\hline Direct bilirubin & & 0.016 & & 0.004 \\
\hline High & reference & & reference & \\
\hline Low & $0.326(0.13-0.81)$ & & $0.387(0.20-0.74)$ & \\
\hline Indirect bilirubin & & 0.000 & & 0.000 \\
\hline High & reference & & reference & \\
\hline Low & $0.073(0.03-0.22)$ & & $0.121(0.05-0.28)$ & \\
\hline
\end{tabular}

volumes of the groups with high TBIL and IBIL levels were smaller compared to the groups with low TBIL and IBIL. This significant difference is $\mathrm{p}=0.037$ for both TBIL and IBIL groups, while $\mathrm{p}=0.298$ for DBIL group. We think that bilirubin inhibits the mTOR pathway by altering the activity of the AMPK pathway, thus revealing its antiproliferative effect, and we observed more limited tumour volumes in the patients with high bilirubin.

As shown in our study, it is known that the patients with small tumour volume generally have better survival. In the survival analysis of the cohort stratified with GTVec 128.5 and TBIL 0.565 cut-off values; in the comparison of high TBIL vs low TBIL groups, significantly longer OS was observed in the high TBIL group in the patients with GTV volume above $128.5 \mathrm{cc}$ ( $\mathrm{p}$ $=0.012$ Supplementary Figure 4A). Based on these data, it is possible to say that the slightly increased bilirubin at the time of diagnosis is associated with both better survival and smaller tumour volume. Investigating the potential causes of this situation, there are studies in literature proving that both bilirubin and RT activate the AMPK pathway.

People with mildly high bilirubin within the normal limits have less metabolic and inflammatory diseases, such as lower body mass indices (BMI) (18), improved glucose and lipid profiles (19), and especially a lower prevalence of diabetes mellitus. It has been shown that AMPK, which plays a key role in cellular energy homeostasis, contributes positively to these results with its modulation at high bilirubin levels (14). In addition, it is known that the AMPK pathway is activated by ionizing radiation, and AMPK activation is associated with a good prognosis in breast (20) and colorectal carcinoma (21). In the in-vitro study on breast

analysis of the cohort, which stratified by the cut-off value that determined for Gtvec; the survival of patients whose GTVec volume exceeded $128.55 \mathrm{cc}$ was 19.2 months, while the survival of patients below $128.55 \mathrm{cc}$ was found to be 37.8 months ( $\mathrm{p}=$ 0.023).

In Chi-Square analysis between the groups with high TBIL, DBIL, and IBIL levels and groups with low TBIL, DBIL and IBIL levels; no significant difference was observed between the groups in terms of age, gender, histology subtype, T stage, N stage, TNM stage, and ChT regimen, except the GTV cc volume. At the time of diagnosis, it was statistically significant that the GTV cc cancer, it has been proven that metformin, which acts with the activation of the AMPK pathway and is evaluated as an antineoplastic agent, increases radiosensitivity by affecting intracellular reactive oxygen radicals (ROS) (20). AMPK is an evolutionarily conserved cellular energy sensor from the serine-threonine kinase family of enzymes found in almost all eukaryotic cells. The high Adenosine Monophosphate (AMP)/Adenosine Triphosphate (ATP) ratio activates AMPK, which inhibits energy-consuming processes and triggers ATP-generating catabolic events to restore energy homeostasis within the cell. Metformin and polyphenols used in diabetes treatment, and exercise activate $\operatorname{AMPK}(22,23)$. 


\section{0-109}

Recently, AMPK has been shown to participate in signalling pathways that respond to genomic stress and regulate cell survival. Apart from regulating intracellular energy, AMPK also plays a key role in the regulation of cancer cell growth and checkpoint control. AMPK inhibits the mammalian target of rapamycin (mTOR) pathway, which is considered a cell growth control signal, by phosphorylating Tuberous Sclerosis protein 2 (TSC2) and regulatory-associated protein of mTOR (Raptor). It also provides cell checkpoint control by playing a role in $\mathrm{p} 53$ phosphorylation and makes cells more susceptible to apoptosis with mitochondrial p53 accumulation. In addition, radiotherapyinduced double-strand DNA breaks (DSB) lead to activation of the kinase Ataxia Telangiectasia Mutated (ATM), which responds through auto-phosphorylation and activation of DNA repair pathways (23). In addition, it is known that activated ATM inhibits mTOR due to AMPK over p53 (24). AMPK activated following RT works to suppress mTOR activation. AMPK activated following RT exposure tries to suppress mTOR activation. In the in-vitro study, it was shown that radiation activates AMPK in lung, prostate and breast cancer and contributes to G2/M phase regulation, and when metformin is added, it has been observed that AMPK activation increases with radiation (23). When AMPK activity was inhibited, it was found to be associated with an increased mTOR activation and survival of cells. In the study conducted in NSCLC cell lines (25), it was shown that metformin, an AMPK activator, made cells more sensitive to radiation and had a synergistic effect in the group, where metformin and radiation were administered together. In addition, NSCLC cells have been shown to be more sensitive to AMPK activation than prostate and breast cancer cells (25). In another study performed on the NSCLC cell line, it was observed that metformin and cisplatin had a radiosensitizer effect, but when the AMPK inhibitor was added, the radiosensitization disappeared. Thus, it has been shown that the radiosensitizing effect is through an AMPK-dependent pathway (26). As shown in our study, even if patients had high tumor volumes, higher bilirubin was associated with a better survival.

Based on this, also in our study, we think that high bilirubin levels increase the activation of AMPK with an additive effect together with RT by making a metformin-like effect. Thus, high bilirubin levels at the time of diagnosis both limited tumour formation in the patients and provided a longer survival in these patients by showing an inhibitory effect on tumour progression with radiotherapy.

\section{Conclusion}

There are some limitations to be mentioned in this study. One of these limitations is that the study is retrospective, hence the potential for bias. Patients were assumed to have increased AMPK activation; we do not have quantitative evidence to support this assumption. Another limitation is that although the patients with standard 60 Gy RT dose were included in this study, ChT regimens are different from each other. In order to reveal the effects of antioxidant effects of bilirubin on $\mathrm{ChT}$ and $\mathrm{RT}$ response and its possible predictive effects on survival, larger studies are needed that take into account the genetic factors of the patients.

\section{References}

1. Siegel RL, Miller KD, Jemal A. Cancer statistics, 2020. CA Cancer J Clin 2020; 70 (1): 7-30.

2. Howlader N, Noone AM, Krapcho M et al. SEER Cancer Statistics Review, 1975-2016, National Cancer Institute. Bethesda, D, https: //seer. cancer.gov/csr/1975_2016/, based on November 2018 SEER data submission, posted to the SEER web site, April 2019.

3. Warde P, Payne D. Does thoracic irradiation improve survival and local control in limited-stage small-cell carcinoma of the lung? A meta-analysis. J Clin Oncol 1992; 10 (6): 890-895.

4. Ettinger DS, Wood DE, Aisner DL et al. NCCN Guidelines Insights: Non-Small Cell Lung Cancer, Version 2.2021. J Natl Compr Canc Netw 2021; 19 (3): 254-266.

5. Vítek L, Schwertner HA. The heme catabolic pathway and its protective effects on oxidative stress-mediated diseases. Adv Clin Chem 2007; 43: $1-57$.

6. Vítek L. The role of bilirubin in diabetes, metabolic syndrome, and cardiovascular diseases. Front Pharmacol 2012; 3: 55.

7. Vítek L. Úloha bilirubinu v prevenci kardiovaskulárních a nádorových onemocnění (Role of bilirubin in the prevention of cardiovascular diseases and cancer). Cas Lek Cesk 2016; 155 (2): 10-14.

8. Liu Y, Li P, Lu J et al. Bilirubin possesses powerful immunomodulatory activity and suppresses experimental autoimmune encephalomyelitis. J Immunol 2008; 181 (3): 1887-1897.

9. Xi XX, Wang HL, Chen T, Dai JR, Hou SY, Chen YG. Prognostic value of preoperative serum bilirubin levels in ovarian cancer. Am J Transl Res 2020; 12 (5): 2267-2280.

10. Horsfall LJ, Burgess S, Hall I, Nazareth I. Genetically raised serum bilirubin levels and lung cancer: a cohort study and Mendelian randomisation using UK Biobank. Thorax 2020; 75 (11): 955-964.

11. Song Q, Wang B, Wang S, Zhang YM, Chen WH. Lower serum levels of bilirubin in the newly diagnosed lung cancer patients: A casecontrol study in China. J Cancer Res Ther 2015; 11 (Suppl 2): C168-C172.

12. Corral-Jara KF, Trujillo-Ochoa JL, Realpe M et al. Conjugated Bilirubin Differentially Regulates CD4+ T Effector Cells and T Regulatory Cell Function through Outside-In and Inside-Out Mechanisms: The Effects of HAV Cell Surface Receptor and Intracellular Signaling. Mediators Inflamm 2016; 2016: 1759027.

13. Haga Y, Tempero MA, Zetterman RK. Unconjugated bilirubin inhibits in vitro cytotoxic T lymphocyte activity of human lymphocytes. Biochim Biophys Acta. 1996; 1317 (1): 65-70.

14. Mölzer C, Wallner M, Kern C et al. Features of an altered AMPK metabolic pathway in Gilbert's Syndrome, and its role in metabolic health. Sci Rep 2016; 6: 30051.

15. Amin MB, Edge S, Greene $\mathbf{F}$ et al. AJCC Cancer Staging Manual (8th edition). Springer International Publishing: American Joint Commission on Cancer; 2017

16. Li N, Xu M, Cai MY et al. Elevated serum bilirubin levels are associated with improved survival in patients with curatively resected non-smallcell lung cancer. Cancer Epidemiol 2015; 39 (5): 763-768. 
17. Jeremić B, Miličić B, Milisavljevic S. Clinical prognostic factors in patients with locally advanced (stage III) nonsmall cell lung cancer treated with hyperfractionated radiation therapy with and without concurrent chemotherapy: single-Institution Experience in 600 Patients. Cancer 2011; 117 (13): 2995-3003.

18. Wallner M, Bulmer AC, Mölzer $\mathbf{C}$ et al. Haem catabolism: a novel modulator of inflammation in Gilbert's syndrome. Eur J Clin Invest 2013; 43 (9): 912-919.

19. Wallner M, Marculescu R, Doberer D et al. Protection from agerelated increase in lipid biomarkers and inflammation contributes to cardiovascular protection in Gilbert's syndrome. Clin Sci (Lond) 2013; 125 (5): 257-264.

20. Zhang Y, Storr SJ, Johnson K et al. Involvement of metformin and AMPK in the radioresponse and prognosis of luminal versus basal-like breast cancer treated with radiotherapy. Oncotarget 2014; 5 (24): 1293612949.

21. Tokunaga R, Cao S, Naseem M et al. AMPK variant, a candidate of novel predictor for chemotherapy in metastatic colorectal cancer: A meta-analysis using TRIBE, MAVERICC and FIRE3. Int J Cancer 2019; 145 (8): 2082-2090.
22. Sanli T, Steinberg GR, Singh G, Tsakiridis T. AMP-activated protein kinase (AMPK) beyond metabolism: a novel genomic stress sensor participating in the DNA damage response pathway. Cancer Biol Ther 2014; 15 (2): 156-169.

23. Sanli T, Rashid A, Liu $\mathbf{C}$ et al. Ionizing radiation activates AMPactivated kinase (AMPK): a target for radiosensitization of human cancer cells. Int J Radiat Oncol Biol Phys 2010; 78 (1): 221-229.

24. Budanov AV, Karin M. p53 target genes sestrin1 and sestrin 2 connect genotoxic stress and mTOR signaling (published correction appears in Cell 2009 Jan 23; 136 (2): 378). Cell 2008; 134 (3): 451-460.

25. Storozhuk Y, Hopmans SN, Sanli T et al. Metformin inhibits growth and enhances radiation response of non-small cell lung cancer (NSCLC) through ATM and AMPK. Br J Cancer 2013; 108 (10): 2021-2032.

26. Riaz MA, Sak A, Erol YB et al. Metformin enhances the radiosensitizing effect of cisplatin in non-small cell lung cancer cell lines with different cisplatin sensitivities. Sci Rep 2019; 9 (1): 1282.

Received August 17, 2021. Accepted September 21, 2021. 